\title{
Sex Equality in Marvel Movies
}

\author{
K. V. Temuzion
}

Assistant Professor of English, Parala Maharaja Engineering College, Berhampur, Odisha, India

"A man of quality is never threatened by a woman of equality"

-Thomas Jefferson

The above quote surmises the importance of equality in gender in society. Movie is a great platform to strongly disseminate any sort of message or perspective deep into the society. Billions of viewers enjoy Marvel movies throughout the world. Marvel movies have gifted the world with a handful of superheroes, the fact which needs to be appreciated but how far it has given importance to the fair sex in these adventures is quite a point to ponder over.

With great power comes the great responsibility. Each and every aspect of Marvel movie is thoroughly observed by and talked about throughout the globe. In such case, if they take up the gallant cause of establishing gender equality across the society, we could have seen a different society altogether.

Before hopping into the discussion, we need to know something known as Bechdel test also known as BechdelWallace test.

This test has 3 parts:

1. Two named female characters

2. Have to talk about something

3. That has nothing to do with a man.

This may seem simple but it's surprising that most of the marvel movies failed to qualify this test.

Now let's take the case of each movie

The following ten marvel movies failed to make it to the test.

- The Incredible Hulk: We have only one named woman known as Betty in the whole movie

- Iron Man: We see one scene where Pepper talks to a journalist. But that was about Tony Stark.

- Captain America: The First Avenger: This movie has only one named female character, Peggy Carter. Even though she is portrayed as a strong and independent woman, this movie failed the test.

- The Avengers: This movie has three named female characters. Though they never talk to each other.
- Ant Man: There are only two named female characters in the film and they don't talk to each other.

- Captain America: Civil War: We have three named female characters out of which Natasha and Wanda never get to talk throughout the film. Third character is May Parker but she only talks to Peter Parker and Tony Stark.

- Doctor Strange: There are only two named female characters in the movie. Christine and the Ancient One. They never meet in the film. We don't even know the real name of Ancient One.

- Spider Man: Homecoming: We have three named female characters in the movie. May Parker, Mary Jane and Liz, but they don't get to talk to each other.

- Thor: Ragnarok: This movie has three named female characters. Topaz don't even get enough screentime to qualify. Rest of the two characters Hela and Valkyrie don't talk to each other.

- Spider Man far from home: Though this movie has three female lead characters which includes Peter's aunt as well as his crush, this movie failed to show one proper conversation between any two female characters.

Here are 13 marvel movies which gave some representation to women and passed the test.

- Iron Man 2: With the introduction of Natasha Romanoff, this turned out to be the first marvel film which passed the test. Pepper and Natasha talks about business for a while.

- Thor: Jane and Darcy are two female characters who talk about physics for a bit which enabled movie to pass, yet most of the time they talk about Thor.

- Iron Man 3: Pepper and Maya briefly talk about Maya's work. So it's a pass.

- Thor: The Dark World: This movie has four named female characters. Jane, Darcy, Frida and Sif. Jane talk about science with Darcy and Frida individually. 
- Captain America: Winter Soldier: We have two named female characters Agent Hill and Natasha Romanoff talks about the bullet that killed Fury.

- Guardians of the Galaxy: Two sisters Nebula and Gamora talk about some orb in the beginning of the movie.

- Avengers: Age of Ultron: Natasha does have a brief conversation with Laura about the sex of her baby. Natasha also speaks with some trainer in her flashback memory but she is unnamed, therefore it can't be counted

- Guardians of the Galaxy: Two named female characters Gamora and the Sovereign talk for a while

- Black Panther: This movie has several named female characters and talking among themselves regarding a wide range of issues.

- Avengers: Infinity War: It has a fight between Scarlet Witch, Proxima, Black Widow and Okoye all female characters. Just a minor exchange of words between them and the movie barely passed the test

- Ant Man and the Wasp: This movie also has four named female characters and Hope Pym interacts with her mother Janet van Dyne regarding quantum realm.

- Captain Marvel: The central character of this movie is a woman, a stronger superhero compared to the other avengers and many other woman characters are shown talking about her and other significant events. So this movie qualified with flying colors.

- Avengers: End Game: This movie has several strong female leads, and the sisters Gamora and Nebula talk about various significant issues.

When the world's most successful movie making franchise rolls out nearly half of its movies ignoring the interests of woman, then what can it be considered as? Always it doesn't require only a man or a group of men to save the world. Even a woman is capable of doing the same, provided she is given the initial grounds.

This is not to establish that Bechdel test is too much authentic to check feminine importance. There are many people who claim that this test is nothing but a basic set of arbitrary rules. But the truth is that Bechdel test isn't asking for too much. Actually very ground level demands that woman should have a name and should talk about something other than man. Is it too much to ask? If the answer is yes, then it's time to change our mindset and trigger representation of woman in films as well.

\section{REFERENCES}

[1] 1.Bray Adam and Cink Lorraine (2017, September 5) Ultimate Marvel, Dorling Kindersley publishers

[2] Lee Stan (2014, March 3) Marvel Encyclopedia. Dorling Kindersley Publishers

[3] O'Sullivan Mike and Ohotmu Team (2017, April 18) Marvel Cinematic Universe Guidebook: The Avengers Initiative, Marvel publishers.

[4] Sanderson Peter (2010, June 16) Obsessed with Marvel, Titan Books Ltd. 